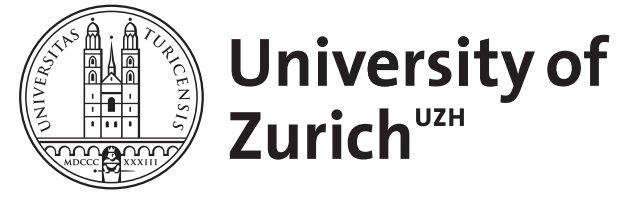

Third Nerve Palsies: Review

Margolin, Edward ; Freund, Paul

DOI: https://doi.org/10.1097/IIO.0000000000000279

Posted at the Zurich Open Repository and Archive, University of Zurich ZORA URL: https://doi.org/10.5167/uzh-174274

Journal Article

Published Version

Originally published at:

Margolin, Edward; Freund, Paul (2019). Third Nerve Palsies: Review. International ophthalmology clinics, 59(3):99-112.

DOI: https://doi.org/10.1097/IIO.0000000000000279 


\section{A Review of Third Nerve Palsies}

\section{Edward Margolin, MD, FRCSC Paul Freund, MD}

\section{- Clinical Vigniette}

A 53-year-old with a history of hypertension reports a 1-day history of a droopy right upper eyelid accompanied by vertical diplopia. On examination, there is partial right upper lid ptosis with impaired levator function and $10 \%$ adduction, $0 \%$ supraduction, and $20 \%$ infraduction on motility testing. Pupils are equal in size, reactive, with no relative afferent pupillary defect.

At the conclusion of this review, you should be able to conclude what should be the next steps in managing this patient.

\section{- General Approach to the Patient With a Third (Oculomotor) Nerve Palsy}

Whenever encountering a patient with any cranial nerve dysfunction, using an anatomic algorithm for localization is a valuable approach. It is important to establish whether a third nerve palsy (TNP) is isolated or other cranial nerves are involved as well and if there are any other neurological symptoms present such as ataxia, tremor, and gait disturbance.

\section{Clinical Pearl}

Most TNPs are caused by microischemia of the nerve due to occlusion/ hyporperfusion of vasa vasorum and are isolated. ${ }^{1}$ In contrast, when TNP is not due to miscroischemia, other neurological signs and symptoms are often present.

In this review, the etiologies of TNPs will be described based on the anatomic location of the lesion (Fig. 1). 


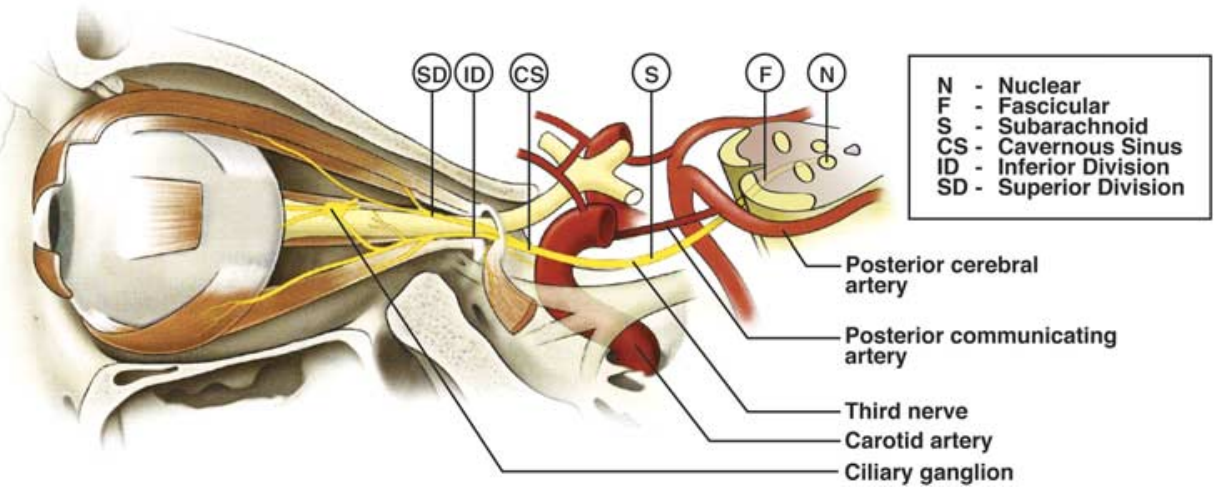

Figure 1. Anatomic course of the third nerve. (Modified from Martin and Corbett. $\left.{ }^{2}\right)\left[\frac{\text { full color }}{0 \mathrm{n} \operatorname{lin\mathrm {e}}}\right.$

When localizing TNP, think of its entire pathway starting with a nucleus in the midbrain, fascicles within the midbrain, the nerve within subarachnoid space traveling under posterior communication artery, entering the lateral wall of the cavernous sinus, reaching superior orbital fissure and innervating each extraocular muscle through a signal transmitted via the neuromuscular junction.

\section{- Overview of Anatomy}

Oculomotor nucleus complex $(\mathrm{OMNC})$ is located in the midbrain ventral to periaqueductal gray matter, extending rostrally towards posterior commissure and caudally towards the trochlear nucleus. ${ }^{3}$ It consists of multiple subnuclei innervating extraocular muscles: levator palpebrae superioris, superior rectus, medial rectus, inferior rectus, inferior oblique muscles and Edinger-Westphal nuclei (EWN) which innervates and the pupillary sphincter and ciliary muscles via parasympathetic fibers.

OMNC is subdivided into 3 larger subnuclei: central subnucleus, lateral subnucleus, and EWN. ${ }^{4}$ Central subnucleus contains medial subnuclei, which innervates the contralateral superior rectus muscle, and a single central caudal subnucleus, which innervates both levator palpebrae superioris. ${ }^{5,6}$

\section{Clinical Pearl}

Nuclear third nerve palsies may cause bilateral ptosis or isolated bilateral superior rectus palsies (when lesion affects medial subnucleus only). Isolated medial rectus palsy cannot be secondary to the nuclear lesion (there are redundant subnuclei contributing to its innervation). ${ }^{7}$ 
EWN is located dorsal and rostral to OMNC and contains 2 distinct populations of neurons: preganglionic cholinergic neurons projecting to the ciliary ganglion and controlling ciliary and pupillary sphincter muscles and peptidergic neurons projecting centrally with diverse roles in controlling sympathetic pathways, appetite, and stress. ${ }^{8}$

The fascicles of the third nerve exit OMNC ventrally and pass through the red nucleus and medial cerebral peduncle. ${ }^{9-12}$ Parasympathetic fibers are located on the outer aspect of the nerve with somatic fibers traveling deeper within the nerve. The proximal third nerve passes between the posterior cerebral artery and the superior cerebellar artery in the anterior incisural space. ${ }^{13}$ This portion of the nerve is supplied by thalamoperforating arteries from the posterior cerebral arteries. ${ }^{14}$ The nerve then travels inferomedial to the uncus, adjacent to the inferolateral edge of the posterior communicating artery for $\sim 5 \mathrm{~mm} .{ }^{13}$ It passes over the posterior petroclinoid fold and pierces the dura, the main fixation point of the nerve to enter the cavernous sinus. ${ }^{15,16}$

In the distal cavernous sinus or proximal superior orbital fissure, the nerve divides into superior and inferior divisions. ${ }^{17}$ The 2 divisions enter the orbit via superior orbital fissure within the annulus of Zinn. ${ }^{18}$ Superior division enters the orbit below the insertion of the superior rectus muscle and innervates inferior aspects of both superior rectus and levator palpebrae superioris. Inferior division enters the orbit adjacent to the lateral rectus before giving off branches to medial and inferior recti muscles. Parasympathetic fibers branch off to synapse in the ciliary ganglion located near the lateral aspect of the optic nerve 2 to $3 \mathrm{~mm}$ posterior to the globe. ${ }^{19}$ Postganglionic reaching the pupillary sphincter and ciliary muscles. The terminal branch of the inferior division innervates inferior oblique on its inferomedial surface.

\section{- Etiologies of Third Nerve Palsies}

Several reports have examined the most common etiologies causing TNPs. In 1 population-based study, the incidence of TNP was found to be 4.2 per 100,000 patients. ${ }^{1}$ Of 145 cases of TNP in 1 US county over 37 years, the most common cause was microvascular (42\%), followed by trauma (12\%), compression by neoplasm (11\%), iatrogenic (postneurosurgery) in $10 \%$ and compression by an aneurysm in 6\%. Most of the aneurysm cases were caused by intracavernous aneurysms which have low rupture rate and are located extradurally with low mortality and morbidity. Only $17 \%$ of patients with microvascular lesions had pupillary involvement, whereas in $64 \%$ of patients with compressive lesions pupil was involved. ${ }^{1}$

In another large retrospective study, 1400 patients with TNPs were examined in a county hospital. ${ }^{20}$ The causative lesion was in subarachnoid 
space in $32 \%$ of patients, cavernous sinus in $23 \%$, brainstem in $14 \%$, nonlocalizing peripheral neuropathy in $18 \%$ and unknown in $13 \%$. The etiology was trauma in $25 \%$ of cases, compression by a tumor in $12 \%$, microischemia in $10 \%$, aneurysmal compression in $11 \%$, postneurosurgery in $10 \%$, stroke in $8 \%$, infection in $5 \%$, and Guillain-Barre syndrome (GBS) in $5 \% .^{20}$ In this study, $53 \%$ of patients with microvascular palsies had pupillary involvement, whereas $98 \%$ of patients with aneurysmal TNPs had involvement of the pupil. ${ }^{20}$

Given that the second study was retrospective and looked at patients in a county hospital only, it is not surprising that trauma was the most likely etiology. This is in contrast to the first study which was populationbased and where microischemic TNPs were most common which probably reflects the practice of most ophthalmologists.

\section{Clinical Pearl}

A minority of TNPs are due to aneurysmal compression thus if no aneurysm is identified on adequate neuroimaging and TNP does not improve after 2 to 3 months, a comprehensive search for other etiologies should be undertaken.

\section{- Nuclear Lesions}

Peculiar anatomy of the third nerve nucleus is responsible for the unique clinical presentations of lesions affecting it in the midbrain. It has been classically taught that patients with nuclear TNP exhibit bilateral complete ptosis, contralateral supraduction deficit and ipsilateral adduction and inferoduction palsies. In practice, all of these findings in the same patient are rare as the causative lesion will almost invariably affect several but not all subnuclei as well as fascicles traversing the substance of the midbrain. ${ }^{21,22}$

\section{Clinical Pearl}

The most common motility deficit in patients with nuclear TNP is adduction palsy. ${ }^{23}$ Pupillary involvement and bilateral ptosis are much less common. ${ }^{23,24}$ Most patients with nuclear TNP will have an accompanying neurological abnormality, most commonly gait disturbance, followed by ataxia and contralateral hemiparesis. Rarely, patients with nuclear TNP might have no other neurological deficits thus brainstem pathology should be kept in the differential diagnosis of patients with isolated TNP and MRI with diffusion-weighted imaging should be obtained if no other etiology is apparent. ${ }^{23,24}$

The most common etiology of the nuclear/fascicular lesions producing TNP would be ischemia in the territory of the medial mesencephalic 
branches of a posterior cerebral artery. Other potential etiologies include neoplasms and inflammatory entities which are much less common.

\section{- Fascicular Syndromes}

The fascicles of the third nerve traverse the midbrain in the dorsal to ventral direction passing through the red nucleus, corticospinal tracts, and near the cerebellar peduncle. When the lesion involves any of these locations, the resulting clinical syndrome will have been well-described. Involvement of the red nucleus leads to contralateral tremor (Nothnagel syndrome), and when the red nucleus and substantia nigra are both affected, contralateral hemiparesis and choreiform movements and tremors may occur (Benedikt syndrome). Corticospinal tract involvement causes contralateral hemiparesis (Weber syndrome). ${ }^{23,24}$

\section{Clinical Pearl}

In patients with midbrain stroke, the most common abnormalities associated with fascicular TNP are a disturbance of standing and gait, as well as ataxia. ${ }^{23,24}$

Ischemia is the most common cause of fascicular TNPs, followed by hemorrhagic and demyelinating lesions. Less common etiologies include neoplasms, inflammatory entities (neurosarcoidosis, Behcet disease, and infections tuberculosis, cysticercosis). ${ }^{25}$

\section{- Subarachnoid Portion}

As the fascicles of the third nerve exit the midbrain ventrally between posterior cerebral artery superiorly and superior cerebellar artery inferiorly, the nerve travels in the subarachnoid space parallel and below posterior communicating artery until it pierces the dura and enters the cavernous sinus.

\section{Clinical Pearl}

The subarachnoid space is the most common anatomic location for the third nerve injury. It is in this segment that is vulnerable to compression, most commonly by an aneurysm arising from the junction of the internal carotid and posterior communicating arteries.

Despite advances in neuroimaging and neurosurgery, patients with subarachnoid hemorrhage produced by a ruptured aneurysm have very high morbidity and mortality rate: $15 \%$ die before reaching the hospital with additional $40 \%$ dying in the hospital and full one third of survivors experiencing major neurological disability. ${ }^{26}$ Thus an unruptured 
cerebral aneurysm producing compressive TNP constitutes a life-threatening emergency.

Even though only a small portion $(\sim 10 \%$ to $15 \%)$ of patients with TNP will harbor an underlying aneurysm, ${ }^{1,20}$ because the cost of missing it is so high, investigations of all patients with TNPs is focused first on detecting an aneurysmal compressive lesion.

One cannot reliably differentiate ischemic and compressive TNP based on the historical/examination findings and thus it is now a fairly wellaccepted standard that all patients with TNPs should undergo noninvasive neuroimaging which if performed using modern equipment and interpreted by a neuroradiologist experienced in vascular imaging, should result in almost $100 \%$ rate of detection of all unruptured aneurysms. ${ }^{27,28}$

Most posterior communicating artery aneurysms must be at least $4 \mathrm{~mm}$ to cause TNP and computed tomography angiography (CTA) and/or magnetic resonance angiography (MRA) should detect all aneurysms that are $\geq 3$ $\mathrm{mm} \cdot{ }^{27-29}$

Other rarer causes of TNP in the subarachnoid space include:

- Compression by the dolichoectatic basilar artery. Although it is very uncommon, it has been reported and careful evaluation of noninvasive angiographic studies should reveal the presence of dilated and tortuous basilar artery. ${ }^{30}$

- Schwannoma of the third nerve which can manifest as recurrent TNP. Imaging may initially be interpreted as normal and symptoms may occur cyclically, however, careful examination of postcontrast magnetic resonance imaging (MRI) images would reveal enhancing nodularity of the third cranial nerve usually in the interpeduncular cistern. ${ }^{31}$

- The third nerve may be affected by the infections in the central nervous system and has been reported in association with tuberculosis, syphilis, Fusobacterium, Streptococcus pyogenes, cysticercosis, Lyme disease, group B streptococcus, Epstein-Barr virus, parvovirus, Cryptococcus, neurobrucellosis, and others. ${ }^{32-42}$

- Compression by neoplasms in the subarachnoid space is an infrequent cause of TNP.

- Infiltration of the nerve by neoplastic cells in subarachnoid space may produce TNP.

- Inflammatory conditions such as neurosarcoidosis and Behcet disease also rarely affect the third nerve in its subarachnoid portion. Of note, as Behcet disease has a predilection for affecting the midbrain, it can produce nuclear/fascicular TNPs as well.

- Expanding cerebral edema and transtentorial herniation may compress the cisternal portion of the third nerve by displaced uncus. ${ }^{43}$

- Severe head trauma usually resulting from high-speed motor vehicle accidents (usually significant enough to produce skull base fractures, facial bone fractures, loss of consciousness) can cause traumatic TNP. These patients almost always develop aberrant regeneration. ${ }^{44}$ 


\section{- Cavernous Portion}

After traversing the subarachnoid space, the third nerve pierces the dura and enters the cavernous sinus where it is located in the lateral dural folds above the fourth nerve and lateral to the intracavernous portion of the internal carotid artery (ICA).

\section{Clinical Pearl}

Because of the unique neurovascular anatomy of the cavernous sinus, TNP may be accompanied by other motor and sensory cranial nerve involvement.

- Most common neoplasms affecting cavernous sinus are meningiomas arising from surrounding structures, craniopharyngiomas, schwannomas usually arising from the branches of the trigeminal nerve but occasionally from the third, fourth and sixth nerves.

- Metastatic lesions can also invade cavernous sinus and produce TNPs. Most commonly they originate from breast, lung, and prostate and by extension of nasopharyngeal squamous cell carcinoma.

- As the cavernous sinus is located lateral to the pituitary gland, expansion of pituitary tumors (most commonly pituitary macroadenomas) or hemorrhage into preexisting adenoma (pituitary apoplexy) can cause the pituitary gland to expand laterally and cause a TNP.

- Most common vascular lesion affecting cavernous sinus (and thus producing TNP) are aneurysms of the intracavernous portion of the ICA which tend to have wide necks, low risk of rupture and low mortality.

- Low flow dural cavernous fistulas cause communication between the small dural branches of the intracavernous ICA and venous filled cavernous sinus. They may cause a TNP and are usually idiopathic being more common in older women with a history of hypertension. Although they can be treated through the endovascular approach, many thrombose and resolve on their own.

- Dural sinus thrombosis (DST) also can affect the third nerve in its cavernous portion. DST can be infectious or noninfectious and cause increased pressure in the cavernous sinus thus causing the dysfunction of any of the cranial nerves passing through it. ${ }^{45}$ Most commonly DST is caused by the spread of infection from the adjacent sphenoid or ethmoid sinuses. Infections involving the face, nose, teeth, tonsils, and soft palate are less common are often encountered. The most common causative organism is Staphylococcus aureus followed by Streptococcus pneumonia, gram-negative bacilli and anaerobes. ${ }^{45}$

- When seeing a diabetic patient with multiple ocular motility deficits of recent onset, one has to consider mucormycosis as a potential source of cavernous sinus infection. Establishing the diagnosis required URGENT examination of the paranasal sinuses by an otolaryngologist. 
- Inflammatory conditions that can affect the cavernous sinus include granulomatous disorders (sarcoidosis, granulomatosis with polyangiitis), infections (usually extending from the nearby sinuses or face), and idiopathic inflammatory syndrome (Tolosa-Hunt syndrome), which is a diagnosis of exclusion after infectious and inflammatory entities have been ruled out.

\section{- Orbital Portion}

As the third nerve exits the cavernous sinus, it splits into superior and inferior divisions, either at the border of the cavernous sinus or as the nerve enters superior orbital fissure. The superior division consists of the fibers innervating superior rectus and levator palpebrae superioris muscles and inferior division contains fibers innervating inferior rectus, the inferior oblique, and medial rectus muscles.

\section{Clinical Pearl}

Knowledge about the split of the third nerve into 2 separate divisions can sometimes help in localizing the lesion when encountering a palsy that only involves muscles innervated by specific division.

In addition to containing the fourth, sixth, and first division of the trigeminal nerves, the superior orbital fissure is located close to the optic canal. This anatomic fact distinguishes superior orbital fissure/cavernous sinus syndrome from orbital apex syndrome (vision loss from optic nerve involvement).

A number of pathologic processes can affect the superior orbital fissure:

- Neoplasms (usually arising from surrounding tissues).

- Infections (including fungal infections).

- Mucocele (usually extending from the neighboring sinuses, most commonly ethmoid sinus as its walls are the thinnest).

- Inflammatory entities (granulomatosis with polyangiitis has a predilection for superior orbital fissure).

- Ocular trauma with resulting orbital wall fractures and hemorrhage.

\section{- Disorders of Neuromuscular Junction}

\section{Clinical Pearl}

Ocular myasthenia gravis (OMG) can sometimes mimic TNP.

Myasthenia does not involve the pupil as it affects acetylcholine receptors on striated muscles only. Other signs can be seen in patients suspected of having OMG include fatigability and variability of symptoms and exam findings. Presence of acetylcholine receptor antibodies has been reported in over $90 \%$ of patients with generalized myasthenia and up to $70 \%$ of patients with ocular myasthenia. ${ }^{46}$ 


\section{- Third Nerve Palsy Associated With Guillain-Barre Syndrome}

\section{Clinical Pearl}

GBS may be associated with multiple cranial neuropathies [Miller Fisher (MF) variant] and even rarer it can be associated with an isolated TNP. ${ }^{47}$

The pathophysiology of cranial nerve palsies in GBS is believed to be peripheral nerve demyelination and most patients have accompanying various degrees of ataxia and areflexia. Ophthalmoparesis is most frequently bilateral. Patients with MF variant of GBS have a higher prevalence of anti-GQ1b antibodies. These antibodies are directed against membrane ganglioside glycoprotein are found in high density on third, fourth, and sixth nerves. ${ }^{48}$ Most patients with MF variant of GBS experience spontaneous recovery which is sometimes hastened by the administration of intravenous immunoglobulins or plasma exchange.

\section{- Congenital Third Nerve Palsy}

\section{Clinical Pearl}

In children, almost half of the cases of TNP are congenital and are presumed to result from embryologic insults, intrauterine damage or birth trauma. ${ }^{49,50}$

Several case reports have described segmental ICA agenesis as the cause of congenital TNP. ${ }^{51,52}$ Absence of internal carotid on 1 side has been postulated to result in an increase of flow through the circle of Willis and increase the risk of aneurysms, including at the junction of ICA and posterior communicating artery, causing compressive TNP. ${ }^{53}$ Clinically congenital TNPs usually present with ptosis and ophthalmoplegia; pupillary involvement is common, and aberrant regeneration is often present. ${ }^{54}$ If there is any suspicion that TNP has not been present at birth but rather developed later, neuroimaging must be performed to rule out compressive causes.

\section{- Aberrant Regeneration}

Also known as oculomotor synkinesis, this phenomenon is due to misdirection of regenerating axons. It occurs in compressive or traumatic TNPs, on average between 2 and 3 months after the onset of compression/ trauma ${ }^{54}$ Because the third nerve has several branches, clinically aberrant regeneration can take make forms. Most commonly observed clinical variant is the elevation of the upper lid on infraduction. Other possibilities include upper lid elevation on adduction or supraduction, pupillary constriction on infraduction or supraduction, globe retraction (presumably from cocontraction of inferior and superior recti muscles) on supraduction, and adduction of the eye on attempted supraduction or infraduction.

www.internat-ophthalmology.com

Copyright (C) 2019 Wolters Kluwer Health, Inc. All rights reserved. 


\section{Clinical Pearl}

Aberrant regeneration signifies a compression or traumatic etiology of TNP and neuroimaging must be performed.

\section{- Rare Causes of Third Nerve Palsy}

- Giant cell arteritis can rarely cause an isolated TNP. All patients over the age of 50 years should be questioned about systemic symptoms of giant cell arteritis and their inflammatory markers should be checked. ${ }^{55}$

- Perineural spread of tumor (usually squamous cell carcinoma of the skin) can rarely cause painful TNP which can be isolated or occur in combination with cranial nerve 2 or 6 dysfunctions. Here MRI of the brain and orbits preferably with FIESTA sequence and/or positron emission tomography scan are most likely to detect perineural spread. ${ }^{56,57}$

- Recurrent painful ophthalmoplegic neuropathy is an entity that was previously known as ophthalmoplegic migraine. ${ }^{58}$ It is characterized by recurrent episodes of $\geq 1$ cranial nerve palsies accompanied by ipsilateral headaches. Isolated TNP accompanied by headache and lasting up to several days with spontaneous resolution and no deficits between the episodes are the most common clinical scenario. All secondary causes should be excluded and detailed neuroimaging, as well as cerebrospinal fluid analysis, should be normal before the diagnosis of recurrent painful ophthalmoplegic neuropathy is made.

- Cyclic oculomotor paresis with spasms a condition that can be congenital or acquired is characterized by oculomotor paresis with continuous episodes repeated every few minutes of lid elevation, eye moving to the midline and pupillary constriction. Episodes (spasms) last 10 to 30 seconds and followed by oculomotor paresis. Congenital cases were thought to be secondary to the lesion of the third cranial nerve nucleus with the retrograde degeneration of the axons, leading to unstable cell membranes with injured nerve generating spontaneous contraction of the muscles innervated by the third nerve. ${ }^{59}$ Acquired cases were described following radiation therapy to the brain and are thought to be variants of ocular neuromyotonia. In acquired cases, the motility is often preserved between the episodes. ${ }^{60,61}$

- Dissection of the intracavernous ICA has been reported as a cause of an isolated TNP. This is presumably due to ischemia resulting from hypoperfusion of inferior hypophyseal and inferior cavernous sinus branches of the intracavernous ICA which supply vasa nervosum to the third nerve in its cavernous portion. ${ }^{62,63}$

\section{Answer to the Clinical Vignette}

- This is a patient with a constellation of findings seen in a partial TNP. www.internat-ophthalmology.com

Copyright (C) 2019 Wolters Kluwer Health, Inc. All rights reserved. 
- Fortunately, current recommendations for the management of any patient with an acute onset of TNP make the job of a clinician easy: the recommendation in this situation is to proceed directly to a noninvasive neuroimaging study.

- CTA is the imaging modality of choice as it a bit more sensitive than MRA in detecting small aneurysms, it is quick to perform and should be readily available.

- It is important that this study is interpreted by an experienced neuroradiologist. ${ }^{27}$ If this study is negative the chance of missing an aneurysmal compression of the third nerve is exceedingly small.

- If CTA is normal and the patient is older (arbitrary: typically pick the age of 50 years or older as of the cutoff) with vascular risk factors, it is reasonable to observe the patient for 2 to 3 months with the presumptive diagnosis of microvascular TNP.

- If microvascular TNP is unlikely, MRI with MRA is the appropriate next step to evaluate the course of the third nerve to exclude other causes.

- If MRI is normal, testing for neuromuscular junction disorders (most commonly myasthenia gravis): acetylcholine receptor antibody titers and single fiber electromyogram.

The authors declare that they have no conflicts of interest to disclose.

\section{- References}

1. Fang C, Leavitt JA, Hodge DO, et al. Incidence and etiologies of acquired third nerve palsy using a population-based method. JAMA Ophthalmol. 2017;135:23-28.

2. Martin TJ, Corbett JJ. Practical Neuro-Ophthalmology. New York, NY: McGraw Hill; 2013.

3. Sharpe J, Wong AM. Anatomy and Physiology of Ocular Motor Systems. In: Miller NR, Newman NJ, Biousse V, Kerrison JB, eds. Walsh and Hoyt's Clinical Neuro-Ophthalmology, 5th ed. Philadelphia, PA: Lippincott Williams \& Wilkins; 2005:809-885.

4. Company V, Moreno-Bravo JA, Perez-Balaguer A, et al. The amniote oculomotor complex. Anat Rec (Hoboken). 2019;302:446-451.

5. Bienfang DC. Crossing axons in the third nerve nucleus. Invest Ophthalmol Vis Sci. $1975 ; 14: 927-931$.

6. Kwon JH, Kwon SU, Ahn HS, et al. Isolated superior rectus palsy due to contralateral midbrain infarction. Arch Neurol. 2003;60:1633-1635.

7. Büttner-Ennever JA. The extraocular motor nuclei: organization and functional neuroanatomy. Prog Brain Res. 2005;151:95-125.

8. Kozicz T, Bittencourt JC, May PJ, et al. The Edinger-Westphal nucleus: a historical, structural, and functional perspective on a dichotomous terminology. J Comp Neurol. 2011;519:1413-1434.

9. Castro O, Johnson LN, Mamourian AC. Isolated inferior oblique paresis from brainstem infarction: perspective on oculomotor fascicular organization in the ventral midbrain tegmentum. Arch Neurol. 1990;47:235-237.

www.internat-ophthalmology.com 
10. Purvin V. Isolated fascicular third nerve palsy. J Neuro-Ophthalmology. 2010;30:263-265.

11. Schwartz TH, Lycette CA, Yoon SS, et al. Clinicoradiographic evidence for oculomotor fascicular anatomy. J Neurol Neurosurg Psychiatry. 1995;59:338.

12. Saeki N, Yamaura A, Sunami K. Bilateral ptosis with pupil sparing because of a discrete midbrain lesion: magnetic resonance imaging evidence of topographic arrangement within the oculomotor nerve. J Neuroophthalmol. 2000;20:130-134.

13. Park HK, Rha HK, Lee KJ, et al. Microsurgical anatomy of the oculomotor nerve. Clin Anat. 2017;30:21-31.

14. Cahill M, Bannigan J, Eustace P. Anatomy of the extraneural blood supply to the intracranial oculomotor nerve. Br J Ophthalmol. 1996;80:177-181.

15. Martins C, Yasuda A, Campero A, et al. Microsurgical anatomy of the oculomotor cistern. Neurosurgery. 2006;58(suppl 2):220-228.

16. Everton KL, Rassner UA, Osborn AG, et al. The oculomotor cistern: anatomy and high-resolution imaging. Am J Neuroradiol. 2008;29:1344-1348.

17. Iaconetta G, De Notaris M, Cavallo LM, et al. The oculomotor nerve: microanatomical and endoscopic study. Neurosurgery. 2010;66:593-601.

18. Gönül E, Düz B, Timurkaynak E, et al. Microsurgical anatomy of the orbital part of oculomotor nerve. Minim Invasive Neurosurg. 2001;44:146-151.

19. Mcdougal DH, Gamlin PD. Autonomic control of the eye. Compr Physiol. 2016;5: 439-473.

20. Keane JR. Third nerve palsy: analysis of 1400 personally-examined inpatients. Can J Neurol Sci. 2010;37:662-670.

21. Ksiazek SM, Slamovitz TL, Rosen CE, et al. Fascicular arrangement in partial oculomotor paresis. Am J Ophthalmol. 1994;118:97-103.

22. Ogawa K, Suzuki Y, Oishi M, et al. Clinical study of twenty-one patients with pure midbrain infarction. Eur Neurol. 2012;67:81-89.

23. Ogawa K, Suzuki Y, Takahashi K, et al. Clinical study of eleven patients with midbrain infarction-induced oculomotor nerve palsy. J Stroke Cerebrovasc Dis. 2016;25:1631-1638.

24. Kim JS, Kim J. Pure midbrain infarction: clinical, radiologic, and pathophysiologic findings. Neurology. 2005;64:1227-1232.

25. Karatas M. Internuclear and supranuclear disorders of eye movements: clinical features and causes. Eur J Neurol. 2009;16:1265-1277.

26. Connolly ES Jr, Rabinstein AA, Carhuapoma JR, et al. American Heart Association Stroke Council; Council on Cardiovascular Radiology and Intervention; Council on Cardiovascular Nursing; Council on Cardiovascular Surgery and Anesthesia; Council on Clinical Cardiology. Guidelines for the management of aneurysmal subarachnoid hemorrhage: a guideline for healthcare professionals from the American Heart Association/American Stroke Association. Stroke. 2012;43:1711-1737.

27. Trobe JD. Searching for brain aneurysm in third cranial nerve palsy. J Neuroophthalmol. 2009;29:171-173.

28. Elmalem VI, Hudgins PA, Bruce BB, et al. Underdiagnosis of posterior communicating artery aneurysm in noninvasive brain vascular studies. J Neuroophthalmol. 2011;31: 103-109.

29. Chaudhary N, Davagnanam I, Ansari SA, et al. Imaging of intracranial aneurysms causing isolated third cranial nerve palsy. J Neuroophthalmol. 2009;29:238-244.

30. Lou M, Caplan LR. Vertebrobasilar dilatative arteriopathy (dolichoectasia). Ann N Y Acad Sci. 2010;1184:121-133.

31. Shin RK, Mejico LJ, Kawasaki A, et al. Transient ocular motor nerve palsies associated with presumed cranial nerve schwannomas. J Neuroophthalmol. 2015;35:139-143.

32. Khalil M, Boncoeur MP, Torny F, et al. Cranial neuritis with enhancement on postcontrast MRI due to Lyme disease. Rev Neurol (Paris). 2003;159:329-331.

33. Spitzer C, Foltys H, Lemmen SW, et al. Bacterial meningitis as a complication of Fusobacterium necroforum infection in adults. Nervenarzt. 2003;74:1118-1121. 
34. Steppberger K, Adams I, Deutscher J, et al. Meningitis in a girl with recurrent otitis media caused by Streptococcus pyogenes-otitis media has to be treated appropriately. Infection. 2001;29:286-288.

35. Ranjith MP, Divya R, Sahni A. Isolated third cranial nerve palsy: a rare presentation of neurocysticercosis. Ir J Med Sci. 2011;180:905-907.

36. Mukherjee S, Askwith JK. Transient isolated oculomotor nerve paralysis in neonatal group B streptococcal meningitis. J Paediatr Child Health. 2008;44:231-232.

37. Erben Y, Gonzalez Hofmann C, Steinmetz H, et al. Isolated neuritis of the oculomotor nerve in infectious mononucleosis. Nervenarzt. 2008;79:462-464.

38. Azran MS, Waljee A, Biousse V, et al. Episodic third nerve palsy with cryptococcal meningitis. Neurology. 2005;64:759-760.

39. Sequeira J, Calado A, Dias M, et al. Parvovirus B19 infection associated with hemolytic anemia and cranial polyneuropathy. J Neurovirol. 2017;23:786-788.

40. Verma R, Patil TB, Lalla R. Disseminated tuberculosis manifesting as pulmonary, meningeal and spinal tuberculosis in an immunocompetent patient. BMJ Case Rep. 2012:2012.

41. Işıkay S, Yılmaz K, Ölmez A. Neurobrucellosis developing unilateral oculomotor nerve paralysis. Am J Emerg Med. 2012;30:2085.

42. Kurihara S, Mori N, Yano H, et al. A case report of syphilitic meningitis with right oculomotor nerve palsy. No To Shinkei. 2003;55:439-442.

43. Maramattom BV, Wijdicks EF. Uncal herniation. Arch Neurol. 2005;62:1932-1935.

44. Elston JS. Traumatic third nerve palsy. Br J Ophthalmol. 1984;68:538-543.

45. Ebright JR, Pace MT, Niazi AF. Septic thrombosis of the cavernous sinuses. Arch Intern Med. 2001;161:2671-2676.

46. Peeler CE, De Lott LB, Nagia L, et al. Clinical utility of acetylcholine receptor antibody testing in ocular myasthenia gravis. JAMA Neurol. 2015;72:1170-1174.

47. St Louis EK, Jacobson DM. Unilateral third nerve palsy caused by Guillain-Barré syndrome. Neurocrit Care. 2004;1:461-464.

48. Schwerer B, Neisser A, Bernheimer H. Distinct immunoglobulin class and immunoglobulin $G$ subclass patterns against ganglioside GQ1b in Miller Fisher syndrome following different types of infection. Infect Immun. 1999;67:2414-2420.

49. Lyons CJ, Godoy F, ALQahtani E. Cranial nerve palsies in childhood. Eye (Lond). 2015;29:246-251.

50. Schumacher-Feero LA, Yoo KW, Solari FM, et al. Third cranial nerve palsy in children. Am J Ophthalmol. 1999;128:216-221.

51. Cherungottil L, Shetty S, Vijayalakshmi P, et al. Congenital oculomotor nerve palsy due to effects of carotid artery agenesis. J AAPOS. 2014;18:507-509.

52. Dhiman R, Gorimanipalli B, Swamy DR, et al. Congenital third nerve palsy associated with midbrain hypoplasia due to bilateral segmental internal carotid artery agenesis. J Neuroophthalmol. 2018;38:483-485.

53. Miller NR. Solitary oculomotor nerve palsy in childhood. Am J Ophthalmol. 1977;83:106-111.

54. Gold DR, Shin RK, Bhatt NP, et al. Aberrant regeneration of the third nerve (oculomotor synkinesis). Pract Neurol. 2012;12:390-391.

55. Thurtell MJ, Longmuir RA. Third nerve palsy as the initial manifestation of giant cell arteritis. J Neuroophthalmol. 2014;34:243-245.

56. Paes FM, Singer AD, Checkver AN, et al. Perineural spread in head and neck malignancies: clinical significance and evaluation with 18F-FDG PET/CT. Radiographics. 2013;33:1717-1736.

57. Ableman TB, Newman SA. Perineural spread of head and neck cancer: ophthalmic considerations. J Neurol Surg B Skull Base. 2016;77:131-139.

58. Huang C, Amasanti M, Lovell B, et al. Recurrent painful ophthalmoplegic neuropathy. Pract Neurol. 2017;17:318-320. 
59. Loewenfeld IE, Thompson HS. Oculomotor paresis with cyclic spasms. A critical review of the literature and a new case. Surv Ophthalmol. 1975;20:81-124.

60. Gadoth A, Kipervasser S, Korczyn AD, et al. Acquired oculomotor nerve paresis with cyclic spasms in a young woman, a rare subtype of neuromyotonia. J Neuroophthalmol. 2013;33:247-248.

61. Miller NR, Lee AG. Adult-onset acquired oculomotor nerve paresis with cyclic spasms: relationship to ocular neuromyotonia. Am J Ophthalmol. 2004;137:70-76.

62. Kogan M, Natarajan SK, Kim N, et al. Third nerve palsy following carotid artery dissection and posterior cerebral artery thrombectomy: case report and review of the literature. Surg Neurol Int. 2014;5(suppl 14):S497-S500.

63. Santos T, Morais H, Oliveira G, et al. Isolated oculomotor nerve palsy: a rare manifestation of internal carotid artery dissection. BMJ Case Rep. 2014:2014. 